\title{
Monitoring of Non Medicinal Treatment of hepatotoxicity induced by Aflatoxin B1 using RAPD
}

\section{Nazar M. Abdalla ${ }^{1}$, Haitham I. El-Mekkawy ${ }^{2}$, Amani A Osman $^{1}$, Osama S. Abdul-Wahab ${ }^{3}$, Al-Ameen N. Mohamed ${ }^{4}$}

${ }^{l}$ College of Medicine, King Khalid University, Saudi Arabia, ${ }^{2}$ College of Science, King Khalid University, Saudi Arabia, ${ }^{3}$ College of Applied Medical Science, King Khalid University, Saudi Arabia, ${ }^{4}$ College of Medicine, Africa International University, Sudan

Abstract:

Background/aim: The RAPD test was performed using 5 sets of primers for liver samples treated with olive oil, black tea, alphatoxin, curcumin, alphatoxin plus black tea, alpha toxin plus curcumin, alphatoxin plus black tea and curcumin. These were compared with untreated samples.

Materials and methods: The RAPD (Random Amplified Polymorphisim DNA) was used to monitor the effect of 4 herbs used to cure animals affected with Asperigellous alpha toxin. Preparation of cDNAs from all treated or untreated controls were used as a template for differential display reaction using different primers in separate reactions.

Results: showed distinct variations in band patterns of treated from untreated tissues using RAPD A2, 4, 5 and 7 primers while the differences in the band patterns obtained by the RAPD 6 primer were low if compared with the other primers. The phylogenetic analysis using the dendrogram for the obtained band pattern of the examined liver samples using different display with 5 different primers showed; the band pattern obtained by differential display grouped of the examined liver samples genotypes into two main clusters.

Conclusion:The study concluded the usefulness of non medicinal treatment of Aflatoxin heptotoxicity monitored by RAPD. 